\title{
Identification and analysis of the $j n k 1$ gene in polyploid hybrids of red crucian carp (Carassius auratus red var.) and common carp (Cyprinus carpio L.)
}

\author{
Y.M. Xiao, M.G. Jiang, Z.W. Luo, Y.H. Zhou, S. Wen, M. Wang, \\ C. Zhang and S.J. Liu
}

Key Laboratory of Protein Chemistry and Developmental Biology of Education Ministry of China, College of Life Sciences, Hunan Normal University, Changsha, China

Corresponding authors: Y.M. Xiao / S.J. Liu

E-mail: yameix@126.com / 1sj@hunnu.edu.cn

Genet. Mol. Res. 13 (1): 906-919 (2014)

Received November 30, 2012

Accepted June 12, 2013

Published February 19, 2014

DOI http://dx.doi.org/10.4238/2014.February.19.1

\begin{abstract}
Jun N-terminal kinase (JNK) is an important member of the mitogen-activated protein kinase superfamily. The allotetraploid crucian carp is a product of distant hybridization of female red crucian carp with male common carp. It is the first natural case of an allotetraploid with stable genetic characters, including fertility of both female and male animals. In this study, 2 jnkl cDNAs (including jnkla and jnk1b) have been cloned from the polyploid crucian carp system, consisting of the allotetraploid crucian carp, the triploid crucian carp, and their original parents (red crucian and common carp). We show that jnkla and jnklb represent 2 splice forms arising from the jnkl gene. On the basis of the genetic structure of jnkla gene in the polyploid crucian carp system, we demonstrated that the allotetraploid crucian carp is phylogenetically closer to its paternal parent (common carp) than to its maternal parent. We further show a similarity between the triploid crucian carp and its original
\end{abstract}


female parent (red crucian carp). Comparisons of genetic structures indicated that the jnklb genes of allotetraploid and triploid crucian carp are more similar to those of the original paternal parent rather than the original female parent (red crucian carp). RT-PCR analysis indicated that both the jnkla and jnklb genes are widely expressed in fish embryos and in the adult organs, displaying distinct features of embryonic-stage and organ specificity in the polyploid crucian carp system.

Key words: $j n k 1$ gene; Polyploidy; Crucian carp; Molecular cloning; Gene expression; Distant hybridization

\section{INTRODUCTION}

The c-Jun N-terminal kinase (JNK) is a member of serine/threonine (Ser/Thr) protein kinases known as the mitogen-activated protein kinase (MAPK) family (Davis, 2000; Kyriakis and Avruch, 2001; Weston and Davis, 2002). In mammalians, JNK-mediated c-Jun induction is necessary for proper cell cycle reentry in UV-irradiated fibroblasts (Davis, 2000; Shaulian et al., 2000; Tournier et al., 2000). By contrast, prolonged JNK activation and c-Jun overexpression can result in apoptosis (Shaulian and Karin, 2002). Our studies suggest that in fish, JNK expression is closely associated with embryonic development and organogenesis, and it plays an important role in ovarian differentiation and development (Xiao et al., 2010).

The diploid crucian carp F1 is a product of the distant hybridization of red crucian carp (Carassius auratus red var., $2 \mathrm{n}=100$, + ) with common carp (Cyprinus carpio L., $2 \mathrm{n}=$ $\left.100,{ }^{\top}\right)$. As an offspring of $F_{1}$, the diploid crucian carp $F_{2}$ has the characteristics of forming diploid gametes. As a result allotetraploid crucian carps $(4 n=200)$ are produced and both allotetraploid females and males are able to breed (Liu et al., 2001a,b). The hybridization of diploid crucian carps to form tetraploid crucian carp takes place naturally without requiring artificial induction treatments. To date, the allotetraploid crucian carp has been stably bred for 19 generations from $F_{3}$ to $F_{21}$. It has been suggested that these allotetraploid crucian carps comprising fertile females and males appear to be the first natural case of an allotetraploid vertebrate animal with stable genetic characters (Li et al., 2002; Sun et al., 2003; Liu, 2010). Therefore, allotetraploid crucian carp is regarded as an indispensable animal model for investigating the origin and the evolution of fish polyploidization in nature. With the allotetraploid crucian carp as a male parent and the red crucian carp as a female parent, triploid hybrids (triploid crucian carp, $3 n=150$ ) have been produced in our lab (Shen et al., 2006; Chen et al., 2009). It is believed that because of their fast growth, improved disease resistance, and culinary value, the triploid crucian carps outperform other kinds of fish (Chen et al., 2009). Therefore, the triploid crucian carp has become a popular breeding fish in China.

Distant hybridizations are involved in species formation and facilitate radiating evolution, and it is suggested that such hybridization causes heterologous polyploidy, a potential source of the formation of new species (Mallet, 2007). It has also been noted that via distant hybridization, the genome of one species may be transferred into another one, resulting in phenotypic and genotypic changes in the resultant progenies.

However, little is known about the changes in genetic structure due to distant hybridization in polyploid hybrids. In this study, we cloned the cDNA of the jnkl gene in the polyploid 
crucian carp system, represented by the allotetraploid crucian carp, the triploid crucian carp, and their original parents, red crucian and common carps. Two subfamilies of the jnkl gene, named $j n k l a$ and jnklb genes, were obtained, and the sequences of jnkla and $j n k l b$ were determined at the cDNA and amino acid levels. Finally, we also studied the expression patterns of the jnkla and jnklb genes during embryonic and adult development of polyploid hybrids.

\section{MATERIAL AND METHODS}

\section{Animal materials}

The allotetraploid crucian, triploid crucian, red crucian, and common carps were obtained from the Engineering Research Center of Polyploid Fish Breeding in Hunan Normal University, Ministry of Education, China.

\section{Molecular cloning of the full-length jnk1a gene}

Total RNA was extracted from gonads of the polyploid crucian carp system following the protocol of Xiao et al. (2010). A reverse transcription-linked polymerase chain reaction (RT-PCR) cloning technique was used to clone the full-length $j n k l$ cDNA from the allotetraploid crucian carp, the triploid crucian carp, and their original parents (red crucian and common carps). Two jnk1a-P1 oligo primers (see Table 1) were designed to amplify a conserved fragment of $719 \mathrm{bp}$ of the cDNA near the 3'-end of the jnkl-coding region by using the goldfish (C. auratus) jnkl gene (GenBank accession No. EU374209). RT-PCR was conducted with a kit from Invitrogen with $2 \mu \mathrm{g}$ total RNA; $2 \mu \mathrm{L}$ RT reaction mixture was mixed with $1 \mu \mathrm{L} 10$

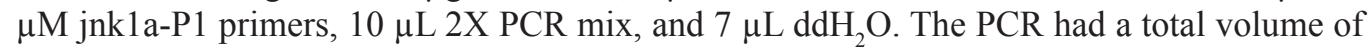
$20 \mu \mathrm{L}$ and was run under the following conditions: 1 hold at $94^{\circ} \mathrm{C}$ for $5 \mathrm{~min}$, followed by 30 cycles of $94^{\circ} \mathrm{C}$ for $30 \mathrm{~s}, 55^{\circ} \mathrm{C}$ for $30 \mathrm{~s}$, and $72^{\circ} \mathrm{C}$ for $60 \mathrm{~s}$. The amplified products were gelpurified and cloned into PMD18-T vector (TaKaRa) for DNA sequencing.

Table 1. Oligo primers used for jnkl cDNA cloning or RT-PCR analysis.

\begin{tabular}{|c|c|c|}
\hline \multicolumn{2}{|c|}{ Name of oligo primers } & \multirow{2}{*}{$\begin{array}{l}\text { Sequences } \\
\text { 5'-AAACTTAGCCGACCCTTCC-3' }\end{array}$} \\
\hline jnk1a-P1 & Forward & \\
\hline & Reverse & 5'-CGTGCTTGACTCGCCTTC-3' \\
\hline \multirow[t]{2}{*}{ jnk1a-P2 } & Forward & 5'-AACACCTACACGCTGCTGGCATCATAC-3' \\
\hline & Reverse & 5'-TCTGAAGCCCAGTAACATAGTAGTG-3' \\
\hline \multirow[t]{2}{*}{ jnk1a-P3 } & Forward & 5'-TGTATGATGCCAGCAGCGTGTAGGTG-3' \\
\hline & Reverse & 5'-TGAGGTGTGAACACATTCAGTAAGCC-3' \\
\hline \multirow[t]{2}{*}{ jnk1b-P1 } & Forward & 5'-AGAAACTCAGCCGACCTTT-3' \\
\hline & Reverse & 5'-TACAGCCAACAGACCATACAT-3' \\
\hline \multirow[t]{2}{*}{ jnk1b-P2 } & Forward & 5'-CTGATGGATGCCAACCTCTGC-3' \\
\hline & Reverse & 5'-TGACACCGTTTCTCATCCGTTC-3' \\
\hline \multirow[t]{2}{*}{ jnk1b-P3 } & Forward & 5'-GGGACCTTCTGTCTAAAATG-3' \\
\hline & Reverse & 5'-CAAATCCCAAGTGACCAAGA-3' \\
\hline \multirow[t]{2}{*}{ jnk1b-P4 } & Forward & 5'-GTGATTTTGAAAGGTGATCT-3' \\
\hline & Reverse & 5'-CCAGGCAAGGGTGTAGGG-3' \\
\hline \multirow[t]{2}{*}{ jnk1b-P5 } & Forward & 5'-TGCTTTAGAATGTGAAGGTGAGAGA-3' \\
\hline & Reverse & 5'-AGTGCTCTTAACAGTGGCAATGATAT-3' \\
\hline \multirow[t]{2}{*}{ jnk1b-P6 } & Forward & 5'-CCCGCCGAGTGGAGGTGTTTTA-3' \\
\hline & Reverse & 5'-GGAGGTGTTTTATTCCGCACAGCATC-3' \\
\hline \multirow[t]{2}{*}{$\beta$-actin-P } & Forward & 5'-CCGTGACCTGACTGACTACCTC-3' \\
\hline & Reverse & 5'-ATACCGCAAGATTCCATACCC-3' \\
\hline
\end{tabular}


The 3'-RACE was performed using Clontech Universal Primer A Mix (UPM) solution (longer primer: $0.4 \mu \mathrm{M}$ 5'-CTAATACGACTCACTATAGGGCAAGCAGTGGTATCAAC GCAGAGT-3'; shorter primer: $2 \mu \mathrm{M}$ 5'-CTAATACGACTCACTATAGGGC-3') and primer jnk1a-P2 (see Table 1). The PCR was performed in a $20-\mu \mathrm{L}$ reaction mix containing $1 \mu \mathrm{L}$ 3'-cDNA library (Smart Race cDNA Reverse Transcriptase Kit, Clontech Inc.), $1 \mu \mathrm{L}$ mixed primers UPM and jnk1a-P2, and $10 \mu \mathrm{L} 2 \mathrm{X}$ PCR mix. Amplification conditions were: 1 hold at $94^{\circ} \mathrm{C}$ for $5 \mathrm{~min}$, followed by 5 cycles of $94^{\circ} \mathrm{C}$ for $30 \mathrm{~s}$ and $72^{\circ} \mathrm{C}$ for $2 \mathrm{~min} ; 5$ cycles of $94^{\circ} \mathrm{C}$ for $30 \mathrm{~s}, 68^{\circ} \mathrm{C}$ for $30 \mathrm{~s}$, and $72^{\circ} \mathrm{C}$ for $2 \mathrm{~min}$; and 25 cycles of $94^{\circ} \mathrm{C}$ for $30 \mathrm{~s}, 65^{\circ} \mathrm{C}$ for $30 \mathrm{~s}$, and $72^{\circ} \mathrm{C}$ for $2 \mathrm{~min}$. The PCR products were re-amplified using $1 \mu \mathrm{L} 10 \mu \mathrm{M}$ primer jnk1aP2 (Table 1) and Nested Universal Primer A (5'-AAGCAGTGGTATCAACG CAGAGT-3'). The 5'-RACE was performed using the 5-RACE kit. The PCR procedures were the same as described above, using jnk1a-P3 primers (Table 1) and UPM mix solution. Amplified products were gel purified and cloned into PMD18-T vector (TaKaRa) for sequencing.

\section{RT-PCR}

Total RNA was extracted from embryos and tissues of the polyploid crucian carp system, comprising the allotetraploid crucian carp, the triploid crucian carp, and their original parents (red crucian and common carps). The embryos were at different stages (2-cell, multiple-cell, blastula, gastrula, neurula, optical vesicle, brain differentiation, muscle differentiation, heartbeat, eye pigmentation, body pigmentation, and hatching). The extracted tissues included heart, muscle, kidney, liver, ovary, and testis.

About $2 \mu \mathrm{g}$ total RNA was used in a $20-\mu \mathrm{L}$ reaction volume, and $2 \mu \mathrm{L}$ reversetranscription reaction was used in PCRs. In the PCR amplification, $\beta$-actin-P primers were used as controls to determine expression of the $j n k 1$ gene. Both $j n k l$-specific and $\beta$-actin-P primers were added into the same PCR mixture, and the reaction was performed for 30 cycles. The resultant PCR products were separated by agarose gel (1.5\%) electrophoresis and DNA bands visualized and photographed under UV illumination. More than 3 batch materials were chosen for each test group, and for each batch material, experiments were repeated more than 3 times.

\section{RESULTS}

\section{Molecular cloning and sequence analysis of the jnkla gene from the polyploid crucian carp system}

Using gene-specific primers (see Table 1: jnk1a-P1, 2 and 3), the full-length jnkla cDNA was cloned from allotetraploid crucian carp (GenBank accession No. JN542469), triploid crucian carp (JN542468), red crucian carp (JN542467), and common carp (JN257262). The jnkla cDNA gene from allotetraploid crucian carp was $1884 \mathrm{bp}$ long containing an open reading frame (ORF) of $1155 \mathrm{bp}$, coding for 384 amino acids. The full-length cDNA of the jnkla gene was $1425 \mathrm{bp}$ long, containing an ORF of $1140 \mathrm{bp}$ coding for 380 amino acids in triploid crucian carp, $1435 \mathrm{bp}$ containing an ORF of $1140 \mathrm{bp}$ coding for 380 amino acids in red crucian carp, and 1777 bp containing an ORF of 1155 bp coding for 384 amino acids in common carp (Table 2). 
Table 2. Full-length cDNAs of jnk1a from allotetraploid crucian carp (AT), triploid crucian carp (3n), red crucian carp (RC), and common carp (CC).

\begin{tabular}{lccccc}
\hline Sample & Full-length cDNAs (bp) & Coding sequence (CDS) & 5'-UTM & 3'-UTM & Total of amino acids \\
\hline AT & 1884 & $226-1380$ & $0-225$ & $1381-1884$ & 384 \\
3n & 1425 & $213-1355$ & $0-212$ & $1356-1425$ & 380 \\
RC & 1435 & $224-1366$ & $0-223$ & $1367-1435$ & 380 \\
CC & 1777 & $181-1335$ & $0-180$ & $1336-1777$ & 384 \\
\hline
\end{tabular}

We next compared the different jnkla cDNA sequences from the polyploid crucian carp system (allotetraploid crucian carp, triploid crucian carp, and their original parents) and observed a high level of sequence similarity among them (Figure 1). The JNK1a protein sequences from allotetraploid crucian and red crucian carp were $96.8 \%$ identical, and $98.1 \%$ identity was observed between the JNK1a proteins from allotetraploid crucian and common carp, and from triploid crucian and common carp. The JNK1a proteins from triploid crucian carp and red crucian were about $100 \%$ identical.

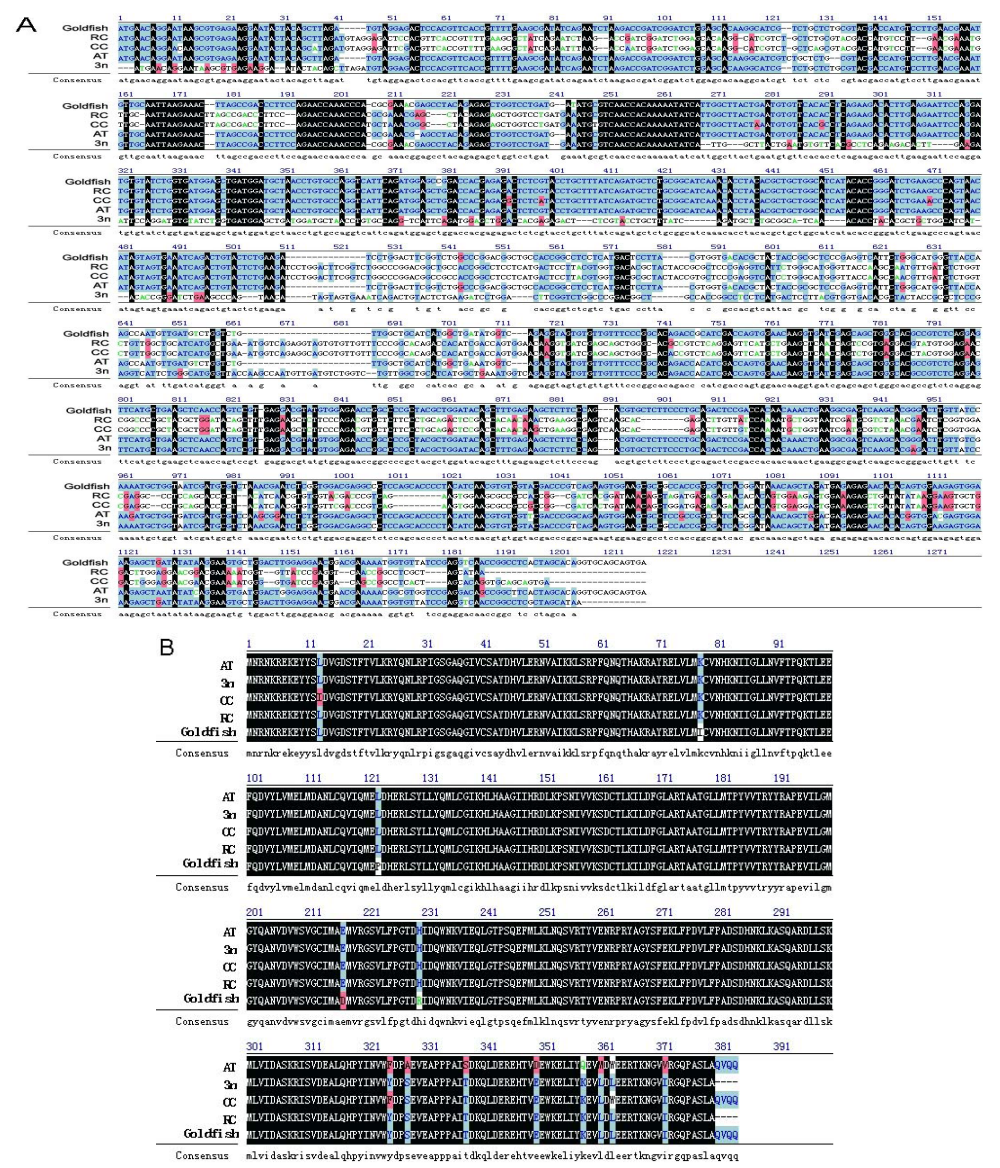

Figure 1. Multiple nucleotide sequence (A) and amino acid sequence alignment (B). Comparison of the amino acid sequences from JNK1 of gold fish and JNK1a of the polyploid crucian carp system [allotetraploid crucian carp (AT), triploid crucian carp (3n), common carp (CC), and red crucian carp (RC)]. 


\section{Expression of the jnkla gene in different tissues and embryos of polyploid crucian carps}

To test whether the jnkla gene may be involved in the development of polyploid crucian carps, the expression of jnkla was analyzed by RT-PCR, by using the primer jnk1a-P1 (see Table 1). As shown in Figure 2, tissues from liver, testis, ovary, brain, kidney, muscle, heart, gill, and eye had high expression levels of the jnkla gene, whereas its expression was very low in the tail fin of the polyploid crucian carps. In allotetraploid crucian carps, the jnkla mRNA levels in the tissues of liver, ovary, kidney, and heart were similar to the jnkla mRNA levels in the same tissues from their original parents (i.e., red crucian carps and common carps). Expression of jnkla in the brain, gill, and eye was lower in allotetraploid crucian carp than in the other 3 crucian carp species. We also noted that in some tissues, such as testis, ovary, brain, heart, and eye, expression of jnkla was higher in the triploid crucian carp than in red crucian, common, and allotetraploid crucian carps. Both triploid crucian carp and red crucian carp displayed jnkla expression in the muscle that was higher than jnkla expression in the muscles of common and allotetraploid crucian carp.

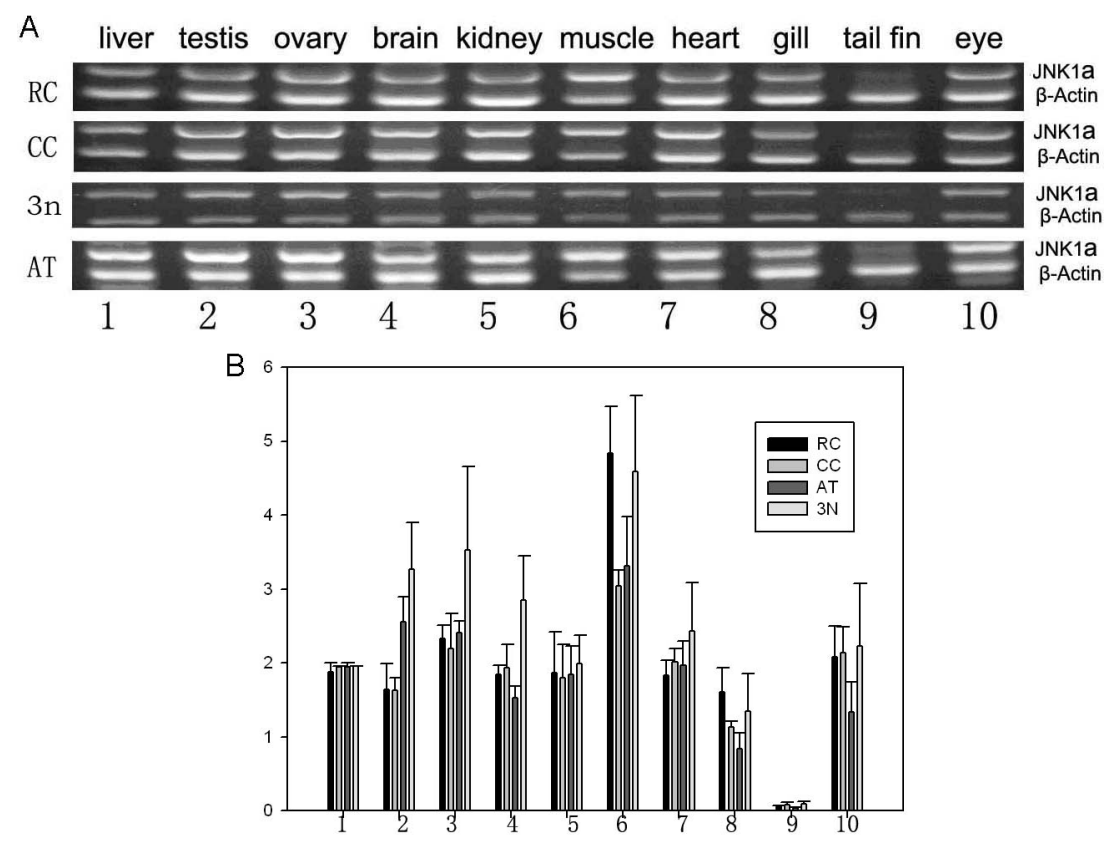

Figure 2. RT-PCR analysis showing the level of the jnkla mRNA in the 10 tissues of the adult fish in the allotetraploid crucian carp (AT), triploid crucian carp (3n), red crucian carp (RC), and common carp (CC) (A). Relative level of expression (fold) calculated by dividing the total pixel from each jnk1 a mRNA band with the total pixel from the corresponding $\beta$-actin mRNA band (B). Quantitative results are from three independent experiments.

During embryonic development of polyploid crucian carps, jnkla expression peaked at the multiple-cell stage, and at the blastula and gastrula stages. In the embryos at the stages of blastula, gastrula, neurula, optical vesicle, eye pigmentation, and hatching, expression of jnkla in triploid crucian carp was distinctly lower than expression in the other 3 crucian carp species (Figure 3). 


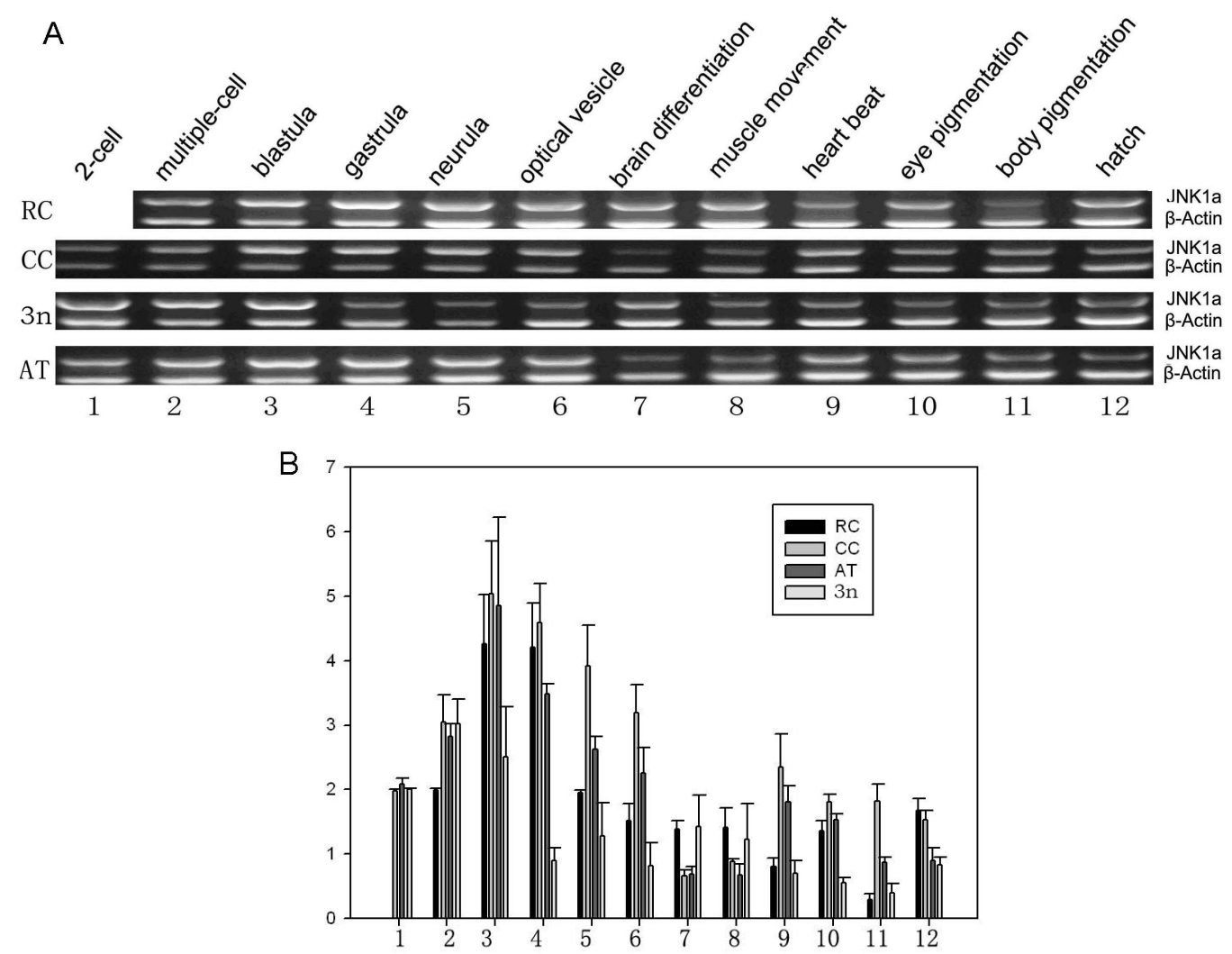

Figure 3. RT-PCR analysis showing the level of the jnkla mRNA in the embryos of allotetraploid crucian carp (AT) and other three fish in the polyploid crucian carp system [triploid crucian carp (3n), common carp (CC) and red crucian carp (RC)] from the stage of cleavage to that of hatching (A). Relative level of expression (fold) calculated by dividing the total pixel from each jnk1a mRNA band with the total pixel from the corresponding $\beta$-actin mRNA band (B). Quantitative results are from three independent experiments.

\section{Molecular cloning of the full-length $j n k 1 b$ cDNA}

During the cloning of the jnkla gene from crucian carps, an additional cDNA sequence was obtained and exactly matched the jnka gene from Japanese carp (Cyprinus carpio, GenBank accession No. is D83273). Therefore, we used the jnka gene sequence of Japanese carp to design 4 primer pairs (jnk1b-P1, 2, 3, and 4) to amplify the cDNA near the 3 '-end of the jnka coding region (see Table 1). The gene-cloning strategy was the same as that for cloning of the jnkla gene described above. As a result, another gene, named jnklb, was obtained from allotetraploid crucian, triploid crucian, red crucian, and common carp.

The full-length cDNA of the $j n k 1 b$ gene had a length of $3601 \mathrm{bp}$ in allotetraploid crucian carp (GenBank accession No. JN542472), 3761 bp in triploid crucian carp (JN542471), 3619 bp in red crucian carp (JN257261), and 3615 bp in common carp (JN542470). All of the $j n k 1 b$ cDNAs from the polyploid crucian carp system contained an ORF of $1284 \mathrm{bp}$, coding for 427 amino acids (Table 3). 
Table 3. Full-length cDNAs of the jnklb gene from the allotetraploid crucian (AT), triploid crucian (3n), red crucian (RC), and common carp (CC).

\begin{tabular}{lccccc}
\hline Sample & Full-length cDNA (bp) & Coding sequence (CDS) & 5'-UTM & 3'-UTM & Total of amino acids \\
\hline AT & 3601 & $215-1498$ & $0-214$ & $1499-3601$ & 427 \\
3n & 3761 & $271-1554$ & $0-270$ & $1555-3671$ & 427 \\
RC & 3615 & $248-1531$ & $0-247$ & $1532-3615$ & 427 \\
CC & 3619 & $237-1520$ & $0-236$ & $1521-3619$ & 427 \\
\hline
\end{tabular}

JNK1b sequences identities were $98.1 \%$ between allotetraploid crucian and red crucian carp, $99.0 \%$ between allotetraploid crucian and common carp, $99 \%$ between triploid crucian and red crucian carp, and $99.5 \%$ between triploid crucian and common carp (see Figure 4 ).

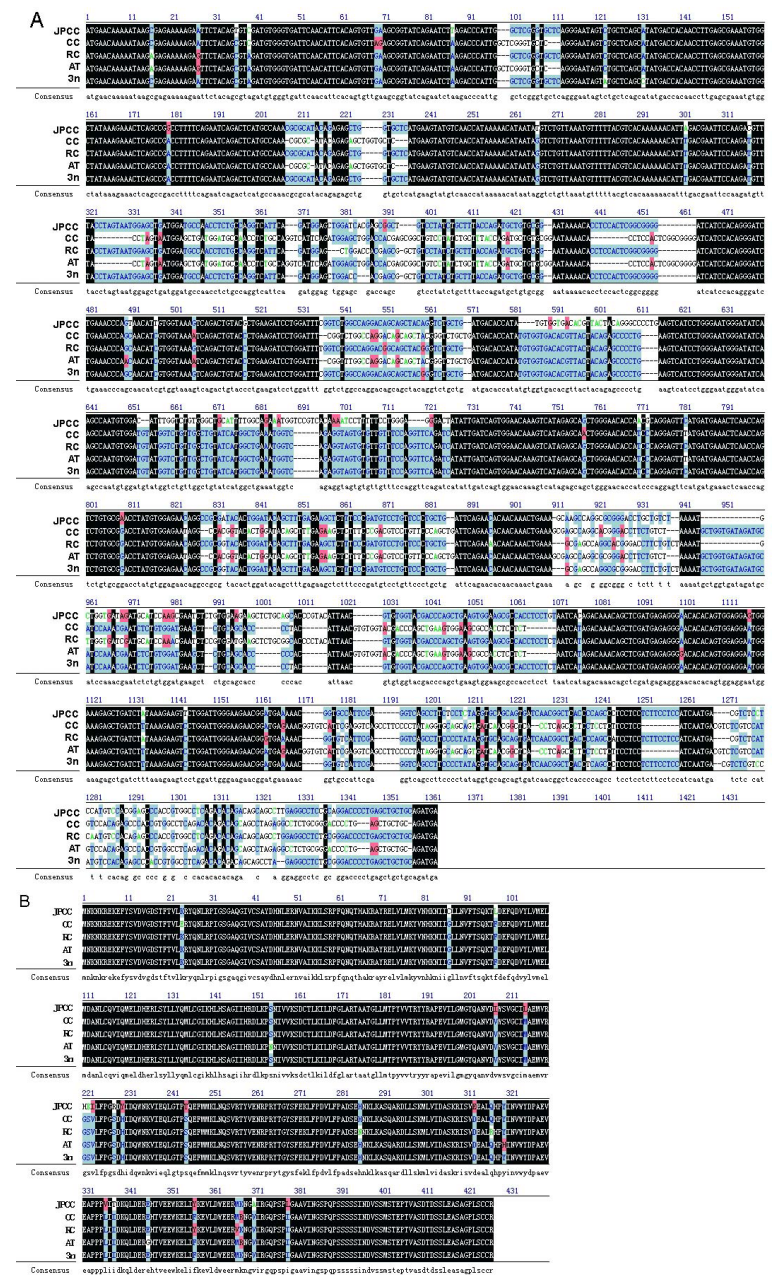

Figure 4. Multiple nucleotide sequence (A) and amino acid sequence alignment (B). Comparison among the JNKa of Japanese carp (JPCC), the JNK1b of allotetraploid crucian carp (AT) and the other three polyploid crucian carps [triploid crucian (3n), red crucian (RC) and common (CC)]. 


\section{Expression of the jnk1b gene in the tissues and embryos of polyploid crucian carps}

jnklb expression was determined in different tissues and embryos of polyploid crucian carps by RT-PCR with primer jnk1b-P1 (Table 10). This analysis indicated high levels of jnklb expression in the tissues of ovary, brain, and eye for all 4 crucian carp species. In contrast, jnklb mRNA levels were very low in the tissues of liver, kidney, heart, gill, and tail fin for all 4 species. In addition, the jnklb gene was more highly expressed in the ovary than in the testis. For both allotetraploid crucian and triploid crucian carp, expression of jnklb in muscle was lower than that in their original parents (see Figure 5).

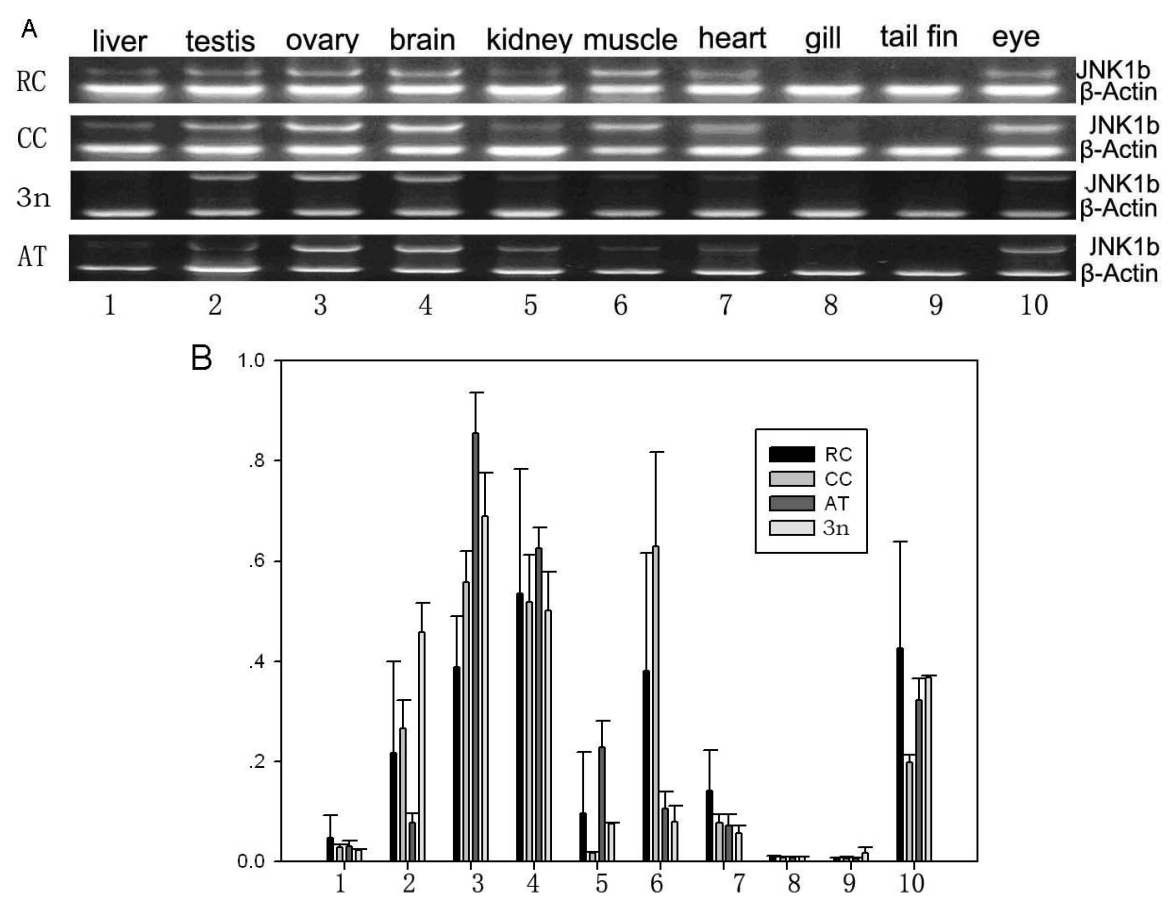

Figure 5. RT-PCR analysis shows that the level of $j n k 1 b$ mRNA in the 10 tissues of the adult fish in the allotetraploid crucian carp (AT), triploid crucian carp (3n), common carp (CC) and red crucian carp (RC) (A). Relative level of expression (fold) was calculated by dividing the total pixel from each jnk1b mRNA band with the total pixel from the corresponding $\beta$-actin mRNA band (B). Quantitative results are from three independent experiments.

In the embryos of the polyploid crucian carp system, expression patterns of the $j n k 1 b$ gene were different from those of the jnkla gene. For example, jnklb expression in the early embryos was higher than in the subsequent stages of embryonic development, where jnklb expression peaked in the early cell-cleavage stage. We also detected strongly upregulated expression of the $j n k 1 b$ gene during brain differentiation in allotetraploid crucian carp, whereas in triploid crucian carp, high expression of jnklb was detected at the stage of muscle development, which was in contrast to jnk1b expression observed in the other 3 crucian carp species (Figure 6). 


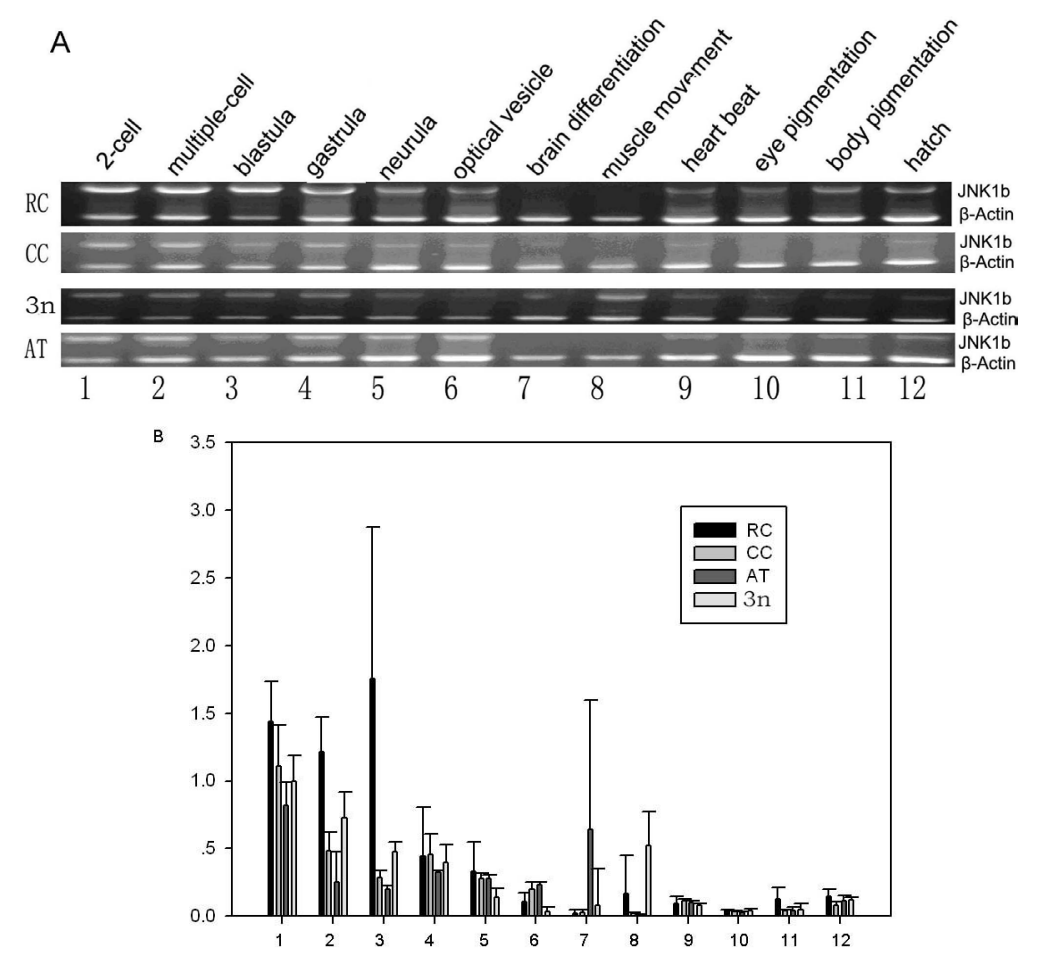

Figure 6. RT-PCR analysis showing the level of jnk1b mRNA in the embryos of allotetraploid crucian carp (AT) and other three fish in the polyploid crucian carp system [triploid crucian carp (3n), common carp (CC) and red crucian carp (RC)] from the stage of cleavage to that of hatching (A). Relative level of expression (fold) calculated by dividing the total pixel from each jnk1b mRNA band with the total pixel from the corresponding $\beta$-actin mRNA band (B). Quantitative results are from three independent experiments.

\section{DISCUSSION}

In recent studies, full-length cDNAs for the $j n k 1$ gene have been cloned in several fish species, including goldfish (GenBank accession No. EU374209) and the rice-field eel (Monopterus albus; EF661977). This has revealed a high level of similarity of the jnkl gene among these fishes and other vertebrates (Xiao et al., 2010). Here, we have cloned the jnkla and jnklb genes from the polyploid crucian carp system, which includes allotetraploid crucian carp, triploid crucian carp, and their parents, red crucian and common carp. An analysis of the genetic distances of the $j n k l$ gene homolog, jnkla, among these different carp species indicated that jnkla maintains a high level of conservation in the polyploid crucian carps (see Figure 2). As shown by the phylogenetic tree constructed from jnkla gene sequences (see Figure 7), triploid crucian carp and its original female parent (red crucian carp) showed a very close phylogenetic relationship, as did allotetraploid crucian and common carp, the original paternal parent of allotetraploid crucian carp. The genetic analysis of the jnkla gene indicated that triploid crucian carp had a closer phylogenetic relationship with its female parent. The jnkla gene analysis also suggested a greater affiliation between allotetraploid crucian carp and its paternal parent. 


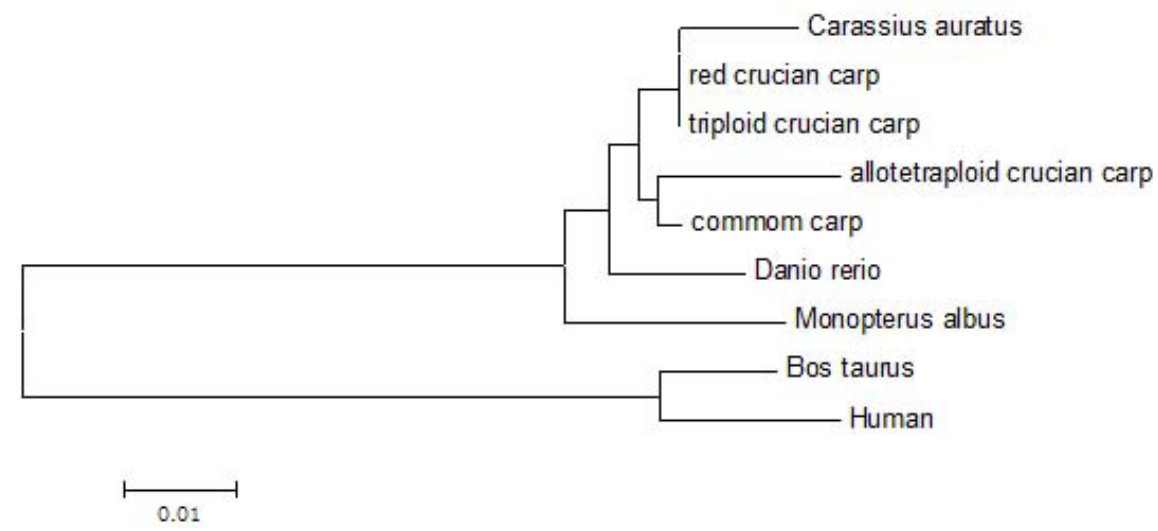

Figure 7. Phylogenetic tree of the jnkla gene in the fish of polyploid crucian carp system (red crucian, common, allotetraploid crucian, and triploid crucian carp) and the jnkl gene of other vertebrates (Carassius auratus, Danio rerio, Monopterus albus, Bos taurus, and human) from NCBI. This phylogenetic tree was generated through comparative analysis of the jnkla (jnkl) coding sequences and using UPGMA calculation and the MEGA3.1 software.

Using the MEGA3.1 software and on the basis of jnklb gene sequences, the genetic distances were determined between allotetraploid crucian, triploid crucian, red crucian, and common carp, and these distances were used to construct a phylogenetic tree by using Neighbor-Joining methods (see Figure 8). In this jnklb gene tree, allotetraploid crucian and the common carp formed a common branch, also shared with triploid crucian carp. Further aggregation with red crucian and Japanese carp led to the formation of a shared clade. These results indicated that the $j n k 1 b$ gene of polyploid crucian carps (i.e., allotetraploid and triploid crucian carp) was more similar to that of their paternal parent than that of the female parent.

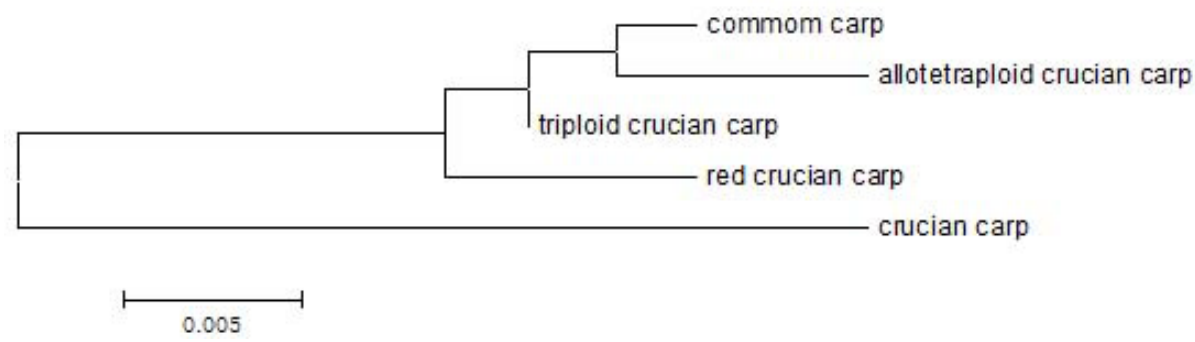

Figure 8. Phylogenetic tree of the jnklb gene in Japanese carp (crucian carp) and in the fish of polyploid crucian carp system (red crucian, common, allotetraploid crucian, and triploid crucian carp). This phylogenetic tree was generated through comparative analysis of the jnklb coding sequences using UPGMA calculation and the MEGA3.1 software.

The MAPK family is a large group of Ser/Thr protein kinases, which are activated by dual phosphorylation of specific Thr and tyrosine (Tyr) residues in a typical "Thr-X-Tyr" motif located within an "activation/phosphorylation loop" (Davis, 1993; Widmann, et al., 1999). As a member of MAPKs, the JNK contains an activation sequence "Thr-Pro-Tyr" (TPY) motif in its kinase domain VIII (Davis, 2000). Three genes, jnk1, jnk2, and jnk3, each encode JNKs 
in mammals (Barr and Bogoyevitch, 2001). Comparisons of these JNK proteins in GenBank, showed that the "TPY" motif appeared in different regions of this protein family: the "TPY" motif appeared at amino acid positions 183-185 in the JNK1 protein, 181-183 in JNK2, and at 221- 223 in JNK3. In this study, we cloned 2 genes jnkla and jnklb, from the polyploid crucian carp system. The identity of jnk1a amino acids sequence in the polyploid crucian carps was $95.5 \%$, and the identity of JNK1b amino acids sequence in the polyploid crucian carps $94.3 \%$. A comparison on the basis of the jnkla and the jnklb gene sequence from the polyploid crucian carp system showed that the amino acid sequences of their predicted proteins were about $83 \%$ similar. The "activation loop" "TPY" motif was presented at positions 183185 sites in all amino acid sequences. The predicted amino acid sequences of the jnklb and the jnkla genes showed changes in about 380-427 sites in the polyploid crucian carp system. These results confirmed that the jnkla and jnklb genes identified here represent 2 splice forms arising from the $j n k 1$ gene in fish.

JNK can phosphorylate a range of substrates owing to its activation by phosphorylation of specific Thr and Tyr within the activation loop. Activated by the upstream kinase MKK4, which phosphorylates Tyr-185 and by MKK7 phosphorylating Thr-183, JNK1 phosphorylates the transcription factor c-Jun on its N-terminal transactivation domain at $2 \mathrm{Ser}$ residues, Ser-63 and Ser-73, to activate AP-1 (Dérijard et al., 1994). Many substrates of JNK are transcription factors, such as Pax2, Elk-1, Foxo4, P53, and ATFII; hence their phosphorylation through JNKs can directly cause changes in gene expression after cells are exposed to a range of cytokines and stress stimuli (Cavigelli et al., 1995; Kawasaki et al., 1996; Mizukami et al., 1997; Chang and Karin, 2001; Tanoue and Nishida, 2003; Fecher et al., 2008). JNK1 also modulates chromatin structure through phosphorylation of histones and regulates different transcription factors (Wolter et al., 2008). In mammals, many diseases are associated with JNK, including eye disease, chronic inflammation (Johnson and Nakamura, 2007), neurodegeneration (Savage et al., 2002), diabetes (Fukuda et al., 2008), and cancer (Uhlirova et al., 2005). The jnkl gene is associated with microtubule. jnkl gene knockout mice exhibition disrupts the formation of anterior commissure tract and generates a progressive loss of microtubules within axons and dendrites (Chang et al., 2003).

JNK signaling is also involved in controlling oocyte development in other vertebrates (Vigneron et al., 2004; Browaeys-Poly et al., 2005). Our previous studies indicated that the expression of the $j n k l$ gene displays distinct patterns during the sex reversal of the rice-field eel M. albus, which has some typical features of natural sexual reversal during its lifecycle (Xiao, 1995; Xiao and Liu, 1995; Xiao et al., 2007). In M. albus, the jnkl gene is highly expressed in the ovary of female individuals, and is substantially decreased at the subsequent intersex stage. However, when the intersex individual develops into the male stage, the expression of JNK1 in the testis is distinctly downregulated (Xiao et al., 2010). In this study, we have demonstrated that both jnkla and jnklb genes show a pattern of wide expression in the embryos and in adult tissues in the polyploid crucian carp system. In comparison with their original parents (red crucian and common carp), distinct expression patterns of $j n k l$ in specific tissues or in adult tissues existed in the allotetraploid crucian and the triploid crucian carp. It was evident that the expression level of jnklb was higher in the ovary than that in the testis of polyploid crucian carps, especially in allotetraploid crucian carp. Conversely, few differences existed in the expression levels of the $j n k l a$ gene among the polyploid crucian carp system, especially for the triploid crucian carp. Fluctuation of jnkl (including jnkla and jnklb) mRNAs during the 
development of polyploid crucian carps implies a fundamental role in regulating the developmental process. The different expression patterns of $j n k l$ genes in the gonads of polyploid crucian carps also suggest an involvement of the jnkl genes in the reproductive development of polyploid hybrid carps, particularly for the fertility of allotetraploid carp and the sterility of triploid carp.

\section{ACKNOWLEDGMENTS}

Research supported by the National Basic Research Program ofChina (\#2012CB22305), the National Science Foundation of China (\#31172399, \#30871908), and the Cooperative Innovation Center of Engineering and New Products for Developmental Biology of Hunan Province.

\section{REFERENCES}

Barr RK and Bogoyevitch MA (2001). The c-Jun N-terminal protein kinase family of mitogen-activated protein kinases (JNK MAPKs). Int. J. Biochem. Cell Biol. 33: 1047-1063.

Browaeys-Poly E, Fafeur V, Vilain JP and Cailliau K (2005). ERK2 is required for FGF1-induced JNK1 phosphorylation in Xenopus oocyte expressing FGF receptor 1. Biochim. Biophys. Acta 1743: 1-4.

Cavigelli M, Dolfi F, Claret FX and Karin M (1995). Induction of c-fos expression through JNK-mediated TCF/Elk-1 phosphorylation. EMBO J. 14: 5957-5964.

Chang L and Karin M (2001). Mammalian MAP kinase signalling cascades. Nature 410: 37-40.

Chang L, Jones Y, Ellisman MH, Goldstein LS, et al. (2003). JNK1 is required for maintenance of neuronal microtubules and controls phosphorylation of microtubule-associated proteins. Dev. Cell 4: 521-533.

Chen S, Wang J, Liu SJ, Qin QB, et al. (2009). The biological characteristics of the improved triploid car. Sci. China Series C Life Sci. 39: 479-484.

Davis RJ (1993). The mitogen-activated protein kinase signal transduction pathway. J. Biol. Chem. 268: 14553-14556.

Davis RJ (2000). Signal transduction by the JNK group of MAP kinases. Cell 103: 239-252.

Dérijard B, Hibi M, Wu IH, Barrett T, et al. (1994). JNK1: a protein kinase stimulated by UV light and Ha-Ras that binds and phosphorylates the c-Jun activation domain. Cell 76: 1025-1037.

Fecher LA, Amaravadi RK and Flaherty KT (2008). The MAPK pathway in melanoma. Curr. Opin. Oncol. 20: 183-189.

Fukuda K, Tesch GH and Nikolic-Paterson DJ (2008). c-Jun amino terminal kinase 1 deficient mice are protected from streptozotocin-induced islet injury. Biochem. Biophys. Res. Commun. 366: 710-716.

Johnson GL and Nakamura K (2007). The c-jun kinase/stress-activated pathway: regulation, function and role in human disease. Biochim. Biophys. Acta 1773: 1341-1348.

Kawasaki H, Moriguchi T, Matsuda S, Li HZ, et al. (1996). Ras-dependent and Ras-independent activation pathways for the stress-activated-protein-kinase cascade. Eur. J. Biochem. 241: 315-321.

Kyriakis JM and Avruch J (2001). Mammalian mitogen-activated protein kinase signal transduction pathways activated by stress and inflammation. Physiol. Rev. 81: 807-869.

Li JZ, Zhang XJ, Liu SJ, Zhou GJ, et al. (2002). Studies on the gonadal development in allotetraploid hybrids of Carassius auratus red var. X Cyprinus carpio L. Acta Hydrobiol. Sin. 26: 116-122.

Liu S (2010). Distant hybridization leads to different ploidy fishes. Sci. China Series C Life Sci. 53: 416-425.

Liu S, Liu Y, Zhou GJ, Zhang XJ, et al. (2001a). The formation of tetraploid stocks of red crucian carp x common carp hybrids as an effect of interspecific hybridization. Aquaculture 192: 171-186.

Liu SH, Cao YC, He XX, Li JZ, et al. (2001b). The formation of tetraploid hybrids of common carp with red crucian carp and the evolutionary significance of teraploidzation in vertebrate. Eng. Sci. China 3: 33-41.

Mallet J (2007). Hybrid speciation. Nature 446: 279-283.

Mizukami Y, Yoshioka K, Morimoto S and Yoshida K (1997). A novel mechanism of JNK1 activation. Nuclear translocation and activation of JNK1 during ischemia and reperfusion. J. Biol. Chem. 272: 16657-16662.

Savage MJ, Lin YG, Ciallella JR, Flood DG, et al. (2002). Activation of c-Jun N-terminal kinase and p38 in an Alzheimer's disease model is associated with amyloid deposition. J. Neurosci. 22: 3376-3385.

Shaulian E, Schreiber M, Piu F, Beeche M, et al. (2000). The mammalian UV response: c-Jun induction is required for exit 
from p53-imposed growth arrest. Cell 103: 897-907.

Shaulian E and Karin M (2002). AP-1 as a regulator of cell life and death. Nat. Cell Biol. 4: E131-E136.

Shen JM, Liu SJ, Sun YD, Zhang C, et al. (2006). A new type of triploid crucian carp- red cruciancarp (†) x allotetraploid (ठ). Progr. Nat. Sci. 16: 1348-1352.

Sun YD, Liu SJ, Zhang C, Li JZ, et al. (2003). The chromosome number and gonadal structure of F9-F11 allotetraploid crucian-carp. Yi. Chuan Xue. Bao. 30: 414-418.

Tanoue T and Nishida E (2003). Molecular recognitions in the MAP kinase cascades. Cell Signal. 15: 455-462.

Tournier C, Hess P, Yang DD, Xu J, et al. (2000). Requirement of JNK for stress-induced activation of the cytochrome c-mediated death pathway. Science 288: 870-874.

Uhlirova M, Jasper H and Bohmann D (2005). Non-cell-autonomous induction of tissue overgrowth by JNK/Ras cooperation in a Drosophila tumor model. Proc. Natl. Acad. Sci. U. S. A. 102: 13123-13128.

Vigneron C, Perreau C, Dupont J, Uzbekova S, et al. (2004). Several signaling pathways are involved in the control of cattle oocyte maturation. Mol. Reprod. Dev. 69: 466-474.

Weston CR and Davis RJ (2002). The JNK signal transduction pathway. Curr. Opin. Genet. Dev. 12: 14-21.

Widmann C, Gibson S, Jarpe MB and Johnson GL (1999). Mitogen-activated protein kinase: conservation of a threekinase module from yeast to human. Physiol. Rev. 79: 143-180.

Wolter S, Doerrie A, Weber A, Schneider H, et al. (2008). c-Jun controls histone modifications, NF-kappaB recruitment, and RNA polymerase II function to activate the ccl2 gene. Mol. Cell Biol. 28: 4407-4423.

Xiao YM (1995). Study on the reproductive biology of Monopterus albus (Zuiew): 2. Female development of Monopterus albus. Acta Sci. Nat. Univ. Norm. Hunan. 18: 45-51.

Xiao YM and Liu Y (1995). Study on the histology in sex changing from intersex to male of Monopterus albus (Zuiew). China J. Fish. 19: 297-301.

Xiao YM, Chen LL, Cheng S, Liu J, et al. (2007).Transformation of the germ stem cells in the gonad development of sex reversal in Monopterus albus. Fen. Zi. Xi. Bao. Sheng Wu Xue. Bao. 40: 196-204.

Xiao YM, Chen L, Liu J, Liu WB, et al. (2010). Contrast expression patterns of JNK1 during sex reversal of the rice-field eel. J. Exp. Zool. B Mol. Dev. Evol. 314: 242-256. 